\title{
Clinical evaluation of CENP-B and Scl-70 autoantibodies in silicosis patients
}

\author{
SUNI LEE ${ }^{1}$, HIROAKI HAYASHI ${ }^{2}$, NAOKO KUMAGAI-TAKEI ${ }^{1}$, HIDENORI MATSUZAKI ${ }^{1}$, KEI YOSHITOME $^{1}$, \\ YASUMITSU NISHIMURA ${ }^{1}$, KOZO URAGAMI ${ }^{3}$, MASAYASU KUSAKA ${ }^{4}$, SHOKO YAMAMOTO $^{1}$, \\ MIHO IKEDA $^{1}$, TAMAYO HATAYAMA ${ }^{1}$, WATARU FUJIMOTO ${ }^{2}$ and TAKEMI OTSUKI ${ }^{1}$ \\ Departments of ${ }^{1}$ Hygiene and ${ }^{2}$ Dermatology, Kawasaki Medical School, \\ Kurashiki, Okayama 701-0192; ${ }^{3}$ Hinase Uragami Iin, Bizen, Okayama 701-3204; \\ ${ }^{4}$ Kusaka Hospital, Bizen, Okayama 705-0021, Japan
}

Received November 24, 2016; Accepted March 16, 2017

DOI: $10.3892 / \mathrm{etm} .2017 .4331$

\begin{abstract}
Silicosis patients (SIL) suffer from respiratory disorders and dysregulation of autoimmunity. Frequent complications such as rheumatoid arthritis, systemic sclerosis (SSc) and vasculitis are known in SIL. Furthermore, we reported previously that some SIL exhibited better respiratory conditions in association with a worse immunological status. In this study, the clinical roles of anti-CENP-B and Scl-70 autoantibodies in SIL were analyzed. The titer index $(\log 10)$ of anti-CENP-B autoantibody in SIL was higher than that of healthy volunteers (HV), and that of SSc was higher than those of HV and SIL. This titer index was positively correlated with an assumed immune status of 1 for HV, 2 for SIL, and 3 for SSc. Moreover, although factor analysis revealed that the titer index of the anti-CENP-B autoantibody formed the same factor with the anti-Scl-70 autoantibody, IgG value and age in SIL cases, another extracted factor indicated that the $\operatorname{Ig} \mathrm{A}$ value and anti-Scl-70 antibody were positively related, but anti-CENP-B showed an opposite pattern in the results of the factor analysis. These findings indicated that the titer index of anti-CENP-B autoantibody may be a biomarker for dysregulation in SIL cases. Future clinical follow-up of SIL may therefore require both respiratory and immunological assessment.
\end{abstract}

\section{Introduction}

Silicosis is one of the typical forms of pneumoconiosis exhibiting pulmonary fibrosis and various pulmonary complications such as lung cancer, tuberculosis, chronic bronchitis, and other ailments (1-3). Patients with silicosis suffer from respiratory symptoms such as hypoxia, cough, sputum and dyspnea. In

Correspondence to: Dr Takemi Otsuki, Department of Hygiene, Kawasaki Medical School, 577 Matsushima, Kurashiki, Okayama 701-0192, Japan

E-mail: takemi@med.kawasaki-m.ac.jp

Key words: silicosis, systemic sclerosis, CENP-B autoantibody, Scl-70 autoantibody addition to these pulmonary problems, silicosis cases (SIL) often exhibit complications of autoimmune diseases such as rheumatoid arthritis (RA, known as Caplan's syndrome) (4-6), systemic lupus erythematosus (SLE) $(7,8)$, anti-neutrophil cytoplasmic antibody (ANCA)-related vasculitis $(9,10)$ and systemic sclerosis (SSc) (11-13).

A consideration of the pathophysiological mechanisms associated with silicosis has led to the conclusion that silica acts as an adjuvant in SIL (14-16). It is from this viewpoint that we have been investigating the direct effects of silica particles on immune competent cells (17-19). Our investigations have shown that silica can chronically activate responder T cells and regulatory $\mathrm{T}$ cells (Treg), resulting in the prolonged survival of responder T cells by suppressing CD95/Fas-mediated apoptosis due to excess production of inhibitors such as soluble Fas molecules with other alternative splicing variants of CD95/Fas and decoy receptor 3 (DcR3) (20-22). In addition, faster loss of Treg due to the excess presence of the CD95/Fas molecule on the cell surface as a result of the chronic activation causes an unbalance of Treg (decrease) and responder $\mathrm{T}$ cells (increase) (23-25).

Moreover, we reported the detection of various auto-antibodies in SIL. For example, the anti-CD95/Fas autoantibody was found in a quarter of SIL cases, and this autoantibody was functional and caused apoptosis of Fas over-expressing cells such as Treg in SIL (26). In addition, anti-topoisomerase I autoantibody (known as anti-Scl-70 autoantibody) was also detected in SIL cases. Our previous study showed that the haplotype HLA-DR15-DQ6 was detected in all four anti-topo I positive Asian Japanese SSc patients that were randomly selected. Furthermore, HLA-DQB1*0402 was identified in three of four anti-topo I positive silicosis patients (27-29). The anti-desmoglein autoantibody was also detected in SIL with no bullous diseases (30). Moreover, anti-caspase 8 autoantibody was detected in SIL (31).

Investigation has also been conducted on the level of soluble interleukin (IL)-2 receptor (sIL-2R) in SIL (32). Although the number of patients in that investigation was low (ten SIL cases), the average level of SIL-2R was between that of healthy volunteers (HV) and SSc. In addition, the levels of sIL-2R in SIL were positively correlated with serum IgG 
and the titer of anti-nuclear antibodies (ANA) as measured by the ELISA method (32). In addition, the results of factor analysis indicated that the sIL-2R level was not extracted with respiratory clinical parameters such as exposure years, $\%$ vital capacity (\%VC) and $\mathrm{V} 25 / \mathrm{H}$ (forced expiratory flow at $25 \%$ vital capacity divided by body height (m)), except for forced expiratory volume $1.0 \mathrm{sec}(\%)$ (FEV1.0\%). However, the sIL-2R level was extracted with other immunological makers such as serum IgG, serum soluble Fas level, and the percentage of $\mathrm{CD} 25+$ in CD4+ cells in peripheral blood mononuclear cells (PBMC). These results indicated that SIL-2R is a maker of immunological activation caused by chronic exposure of silica particles on responder T cells in SIL and is a maker of immune stimulation in SIL (32).

It is on the basis of these aforementioned studies that we measured and analyzed the clinical properties of anti-CENP-B and anti-Scl-70 autoantibodies in SIL.

\section{Materials and methods}

Subjects. All subjects were Japanese. 19 HV [age (median $=46.0$ years old (y.o.): mean \pm standard deviation $(\mathrm{SD})=44.8 \pm 8.6$ y.o.), male: female $(\mathrm{M}: \mathrm{F})=8: 11], 20$ SIL [age (median=73.5 y.o.): mean $\pm \mathrm{SD}=74.9 \pm 5.4)$, male: female $(M: F)=19: 1$ ] and $25 \mathrm{SSc}$ [age (median=65.0 y.o.): mean $\pm \mathrm{SD}=62.3 \pm 12.1)$, male: female $(\mathrm{M}: \mathrm{F})=3: 22]$ were the subjects of this study. All SIL were brickyard workers in Bizen, Okayama, Japan, and were diagnosed according to the ILO 2,000 (33) guideline for pneumoconiosis and clinically followed in Kusaka Hospital or Hinase Uragami Iin according to Japanese law regarding the medical care of pneumoconiosis patients. The amount of free silica inhaled by these patients was estimated to be as high as 40 to $60 \%$ as determined from the work environment. These individuals did not show any symptoms of autoimmune diseases such as sclerotic skin, Raynaud's phenomenon, facial erythema and arthralgia. The SSc patients were diagnosed and monitored by the Dermatology Department, Kawasaki Medical School Hospital, Kurashiki, Japan. This study was approved by the Ethics Committee of Kawasaki Medical School (\#374-2, \#883 and \#912) and samples were obtained only from subjects who provided written informed consent.

Clinical parameters. Serum samples from all subjects were analyzed for ANA using the ELISA-based MESACUP ANA TEST (Medical \& Biological Laboratories, Co., Ltd., Nagoya, Japan), in which there are several recombinant proteins such as RNP, SS-A/Ro, SS-B/La, Scl-70, Jo-1 and Ribosomal P in vitro transcribed U1 RNA and CENP-B protein, and purified antigen (Sm, SS-A/Ro, Scl-70, Histone and DNA). The results were calculated as a titer index according to the following manufacturer's formula:

[Titer index $=\left(\mathrm{A}_{450}\right.$ of sample- $\mathrm{A}_{450}$ of standard serum \#1 (negative control) provided by manufacturer) divided by ( $\mathrm{A}_{450}$ anti-CENP-B antibody standard serum \#2 (positive control)- $\mathrm{A}_{450}$ of standard serum \#1 (negative control) provided by manufacturer)].

Since the ANA assay used in this study including various individual autoantibodies as mentioned above, we specifically extracted the titer index of anti-CENP-B and anti Scl-70 autoantibodies. Since SSc is one of the most frequent autoimmune complications in SIL, we reported the detection of anti-Scl-70 autoantibodies using a different series of SIL patients and had sufficient numbers of comparable SSc samples. In addition, we had reported that detection of anti-Scl-70 auto-antibody in serum of SIL with specific HLA type. Furthermore, serum fron SSc patients were kindly provided for this study, The clinical roles of these autoantibodies in SIL were then evaluated. In addition to these titer indices, serum $\operatorname{IgG}, \mathrm{A}$ and $\mathrm{M}$ were also measured.

The other clinical parameters of SIL that were analyzed included age, exposure years, FEV1.0\%, body-height corrected $\mathrm{V} 25 / \mathrm{H}$ and PR (profusion rate; radiological classification, numbered according to the ILO guideline, 1 to 4). Although the subjective dyspnea rating according to the Hugh-Jones classification (1, slight; 2 , mild; 3 , moderate; and 4, severe) was also recorded, all SIL had a rating of 3 except for one case with a rating of 4 . The dyspnea rating was therefore not used in the subsequent statistical analysis.

Statistical analysis. The $\log _{10}$ values of titer indices of anti-CENP-B and Scl-70 autoantibodies were compared among HV, SIL and SSc because the titer indices are more suitable for evaluation using $\log _{10}$ values instead of actual indices due to the method of sample dilution and the fact that the pathophysiologically higher patients showed logarithmically high titer indices. Comparison of the three groups was performed using the non-parametric Kruskal-Wallis test.

Analysis of correlations between titer indices of both autoantibodies and the assumed immunological status of the three groups $(\mathrm{HV}=1.0, \mathrm{SIL}=2.0$ and $\mathrm{SSc}=3$, as continuous variables) was conducted using the non-parametric Spearman's test.

To assess titer indices of both autoantibodies among SIL cases, we performed individual correlations for each clinical parameter and conducted factor analysis. Correlations were obtained using the non-parametric Spearman's test.

In addition, the factor analysis was performed. Factor analysis is a statistical method used to describe variability among observed, correlated variables in terms of a potentially lower number of unobserved variables called factors. In this study, it was required to assess whether or not the titer value of anti CENP-B as well as anti Scl-70 antibodies in SIL related with respiratory variables, or related with immunological variables. Thus, five assumed respiratory variables (age, PR, $\%$ VC, FEV1.0 (\%) and V25/H) and three other immunological variables (IgG, A and $\mathrm{M}$ ) with above-mentioned titers of two auto-antibodies were applied to the factor analysis.

All statistical analyses were performed using SPSS version 21 (IBM Japan, Tokyo, Japan).

\section{Results}

Comparison of titer indices of anti-CENP-B and anti-Scl-70 autoantibodies in $H V$, SIL and SSc. As shown in Fig. 1, the anti-CENP-B titer index $\left(\log _{10}\right)$ in SIL was significantly higher than that of HV. The titer index in SSc was also significantly higher than that of HV and SIL. However, the titer index of anti-Scl-70 autoantibody was significantly higher in SSc than in HV and SIL. Since the clinical properties 
A

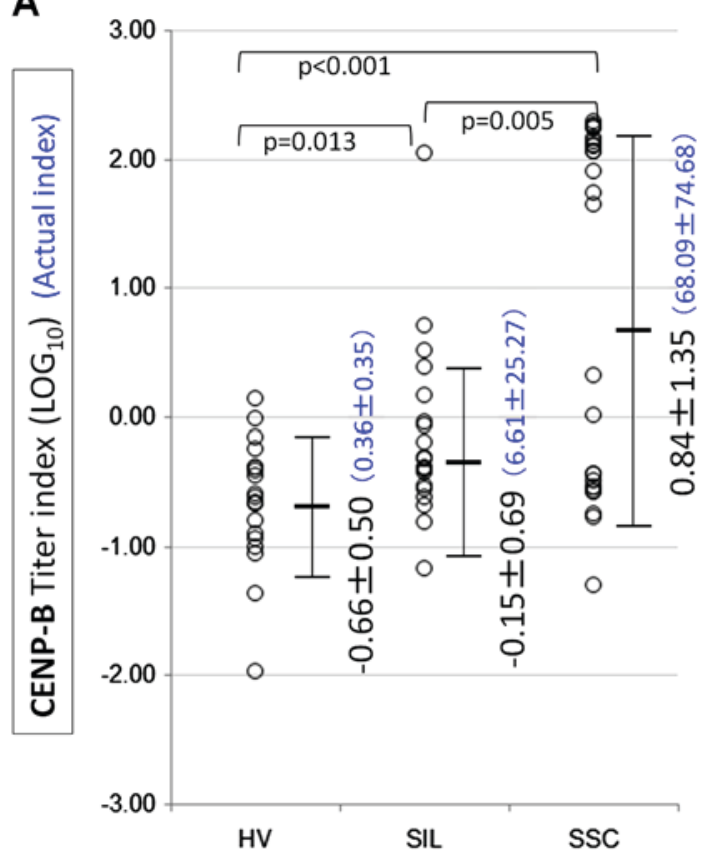

B

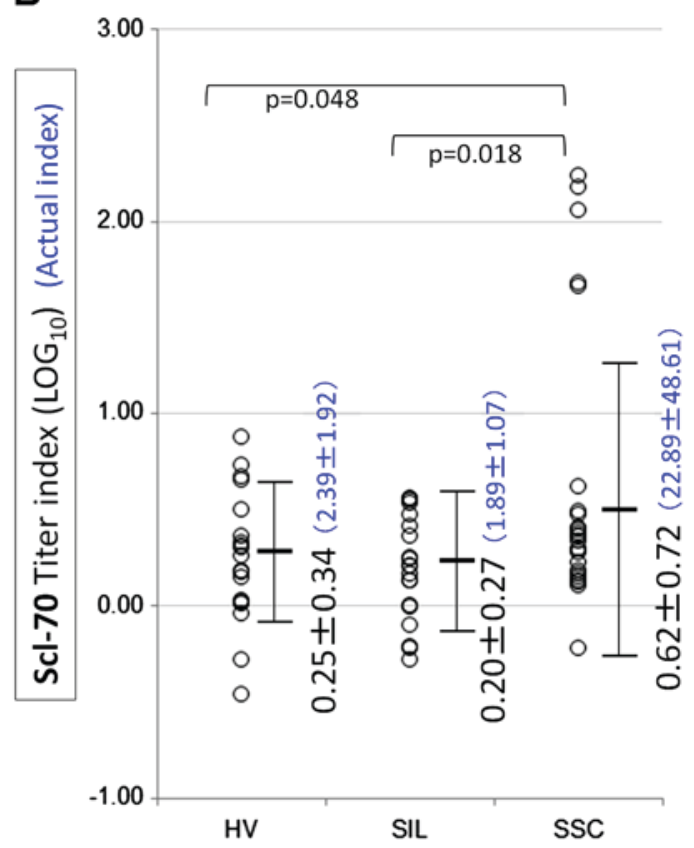

Figure 1. Comparison of titer indices ( $\left.\log _{10}\right)$ and actual indices (in blue) of (A) anti-CENP-B and (B) anti-Scl-70 autoantibodies in healthy volunteers (HV), silicosis patients (SIL) and systemic sclerosis patients (SSc). Statistical comparisons used the non-parametric Kruskal-Wallis test.

A

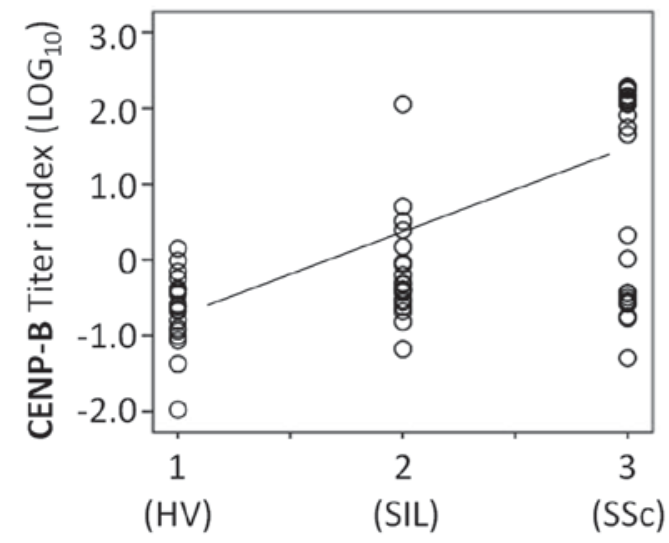

Assumed immune status
B

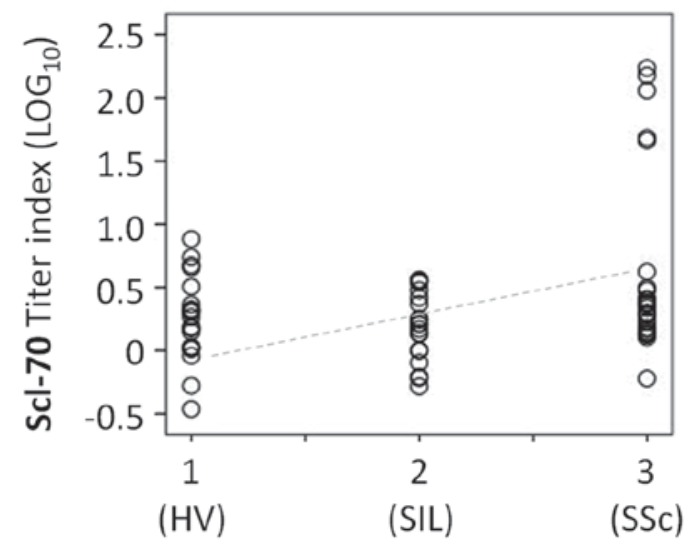

Assumed immune status

$\rho=0.195, p=0.122$

Figure 2. Correlation between titer indices $\left(\log _{10}\right)$ and actual indices (in blue) of (A) anti-CENP-B and (B) anti-Scl-70 autoantibodies and the assumed immune status of 1, 2 and 3 for HV, SIL and SSc, respectively, as the continuous variables. Statistical comparisons used the non-parametric Spearman's test.

of both autoantibodies differ, namely, the anti-CENP-B autoantibody is specifically detected in localized SSc with relatively mild symptoms and CREST syndrome (34), whereas the anti-Scl-70 autoantibody is detected in cases of diffuse SSC with severe visceral lesions such as lung fibrosis [Basu and Reveille (35)], part of the examined SSc cases were positive for both autoantibodies and these cases did not overlap. In addition, only one SIL case showed an equivalent higher titer index compared with positive cases in SSc; however, other SIL also showed a higher tendency compared with HV. This tendency was not found following examination of the anti-Scl-70 autoantibody.
Correlation between titer indices of both autoantibodies and assumed immune status. As mentioned above, if the assumed immune status supposedly progresses in the sequence 1, 2 and 3 for HV, SIL and SSc, respectively, the correlation between the titer index of anti-CENP-B autoantibody and assumed immune status was significantly positive $(\rho=0.548$, $\mathrm{P}<0.001$ ), whereas that between the titer index of anti-Scl-70 autoantibody and assumed immune status was not statistically significant (Fig. 2).

Correlation among various clinical parameters of SIL. To assess the titer indices of both autoantibodies, the individual 
Table I. Correlations among various respiratory and immunological parameters.

\begin{tabular}{|c|c|c|c|c|c|c|c|c|c|c|c|c|c|}
\hline \multirow[b]{2}{*}{ Parameter } & \multirow[b]{2}{*}{ Unit } & \multirow{2}{*}{$\begin{array}{c}\text { ANA } \\
\text { Titer } \\
\text { index }\end{array}$} & \multicolumn{2}{|c|}{ CENP-B Scl-70 } & $\operatorname{IgG}$ & $\operatorname{Ig} \mathrm{A}$ & $\operatorname{IgM}$ & Age & PR & EY & $\% \mathrm{VC}$ & FEV1.0 & $\mathrm{V} 25 / \mathrm{H}$ \\
\hline & & & $\begin{array}{l}\text { Titer } \\
\text { index }\end{array}$ & $\begin{array}{l}\text { Titer } \\
\text { index }\end{array}$ & $\mu \mathrm{g} / \mathrm{ml}$ & $\mu \mathrm{g} / \mathrm{ml}$ & $\mu \mathrm{g} / \mathrm{ml}$ & Years & Grade & Years & $\%$ & $\%$ & $1 / \mathrm{sec} / \mathrm{m}$ \\
\hline \multirow[t]{2}{*}{ ANA } & $\mathrm{CC}$ & & 0.177 & 0.636 & 0.430 & 0.041 & 0.226 & 0.328 & -0.025 & 0.004 & -0.116 & 0.132 & 0.063 \\
\hline & $\mathrm{p}$ & & 0.454 & 0.003 & 0.058 & 0.865 & 0.339 & 0.158 & 0.916 & 0.987 & 0.627 & 0.578 & 0.793 \\
\hline \multirow[t]{2}{*}{ CENP-B } & $\mathrm{CC}$ & & & 0.516 & 0.042 & 0.140 & -0.063 & 0.222 & -0.260 & -0.052 & -0.116 & 0.251 & 0.055 \\
\hline & $\mathrm{p}$ & & & 0.020 & 0.860 & 0.556 & 0.791 & 0.347 & 0.269 & 0.828 & 0.627 & 0.286 & 0.818 \\
\hline \multirow[t]{2}{*}{ Scl-70 } & $\mathrm{CC}$ & 0.636 & 0.516 & & 0.168 & 0.444 & 0.325 & 0.215 & -0.119 & -0.245 & -0.024 & 0.299 & 0.270 \\
\hline & $\mathrm{p}$ & 0.003 & 0.020 & & 0.478 & 0.050 & 0.162 & 0.362 & 0.618 & 0.297 & 0.920 & 0.200 & 0.249 \\
\hline \multirow[t]{2}{*}{$\mathrm{IgG}$} & $\mathrm{CC}$ & & & & & 0.006 & -0.009 & 0.281 & -0.002 & 0.039 & 0.159 & 0.060 & 0.145 \\
\hline & $\mathrm{p}$ & & & & & 0.980 & 0.970 & 0.230 & 0.995 & 0.870 & 0.502 & 0.801 & 0.541 \\
\hline \multirow[t]{2}{*}{$\operatorname{IgA}$} & $\mathrm{CC}$ & & & 0.444 & & & 0.062 & 0.066 & -0.030 & 0.044 & -0.069 & -0.096 & 0.032 \\
\hline & $\mathrm{p}$ & & & 0.050 & & & 0.796 & 0.781 & 0.900 & 0.853 & 0.772 & 0.686 & 0.895 \\
\hline \multirow[t]{2}{*}{ IgM } & $\mathrm{CC}$ & & & & & & & 0.100 & 0.011 & -0.021 & -0.912 & 0.341 & 0.405 \\
\hline & $\mathrm{p}$ & & & & & & & 0.867 & 0.930 & 0.963 & 0.416 & 0.141 & 0.076 \\
\hline \multirow[t]{2}{*}{ Age } & $\mathrm{CC}$ & & & & & & & & 0.024 & 0.183 & -0.315 & 0.057 & 0.049 \\
\hline & $\mathrm{p}$ & & & & & & & & 0.921 & 0.440 & 0.176 & 0.813 & 0.838 \\
\hline \multirow[t]{2}{*}{ PR } & $\mathrm{CC}$ & & & & & & & & & -0.077 & 0.350 & -0.496 & -0.289 \\
\hline & $\mathrm{p}$ & & & & & & & & & 0.748 & 0.130 & 0.026 & 0.216 \\
\hline \multirow[t]{2}{*}{ EY } & $\mathrm{CC}$ & & & & & & & & & & -0.084 & -0.153 & -0.294 \\
\hline & $\mathrm{p}$ & & & & & & & & & & 0.724 & 0.518 & 0.208 \\
\hline \multirow[t]{2}{*}{$\% \mathrm{VC}$} & $\mathrm{CC}$ & & & & & & & & & & & -0.313 & 0.014 \\
\hline & $\mathrm{p}$ & & & & & & & & & & & 0.179 & 0.955 \\
\hline \multirow[t]{2}{*}{ FEV1.0 (\%) } & $\mathrm{CC}$ & & & & & & & & & & & & 0.860 \\
\hline & $\mathrm{p}$ & & & & & & & & & & & & $<0.001$ \\
\hline \multirow[t]{2}{*}{$\mathrm{V} 25 / \mathrm{H}$} & $\mathrm{CC}$ & & & & & & & & & & & 0.860 & \\
\hline & $\mathrm{p}$ & & & & & & & & & & & $<0.001$ & \\
\hline
\end{tabular}

The upper-right part of the table shows all correlations $(\rho=c c$ and $p$ ) among immunological and respiratory parameters in silicosis patients. The lower-left panel represents significant correlations $(\mathrm{P}<0.05)$. Bold font indicates a significant correlation $(\mathrm{P}<0.05)$. CC, coefficient of correlation; p, Significance; ANA, antinuclear antibody; CENP-B, centromere protein B; Scl-70, anti-topoisomerase; Ig, immunoglobulin; PR, profusion rate; EY, exposure years; \%VC, \% vital capacity; FEV1.0, forced expiratory volume 1.0 (sec); V25/H, forced expiratory flow at 25\% vital capacity divided by body height $(\mathrm{m})$.

correlations among the various clinical parameters in SIL were examined. The immunological parameters comprised titer indices of ANA, anti-CENP-B and Scl-70 autoantibodies, serum IgG, A and $\mathrm{M}$. The respiratory parameters comprised age, PR, exposure years, \%VC, FEV1.0\% and $\mathrm{V} 25 / \mathrm{H}$. As shown in Table I, the titer indices of anti-CENP-B and Scl-70 autoantibodies showed a significant positive correlation $(\rho=0.516, P=0.020)$. Although the titer index of the anti-CENP-B autoantibody showed a significant correlation with other parameters examined, the titer index of the anti-Scl-70 autoantibody showed a significant positive correlation with the ANA titer index and serum IgA. Since the measurement of ANA in this study used a multiplex ELISA assay that included the Scl-70 protein, a positive correlation with the ANA titer index may be expected, although the CENP-B protein was also included in the ANA kit without any significant positive correlation.
Factor analysis in SIL. Factor analysis was performed to identify the role of both autoantibodies in contributing to the pathophysiology of SIL and shown in Table II. Factor analysis is usually used to extract unexpected relations among various variables observed or examined in order to form newer insights and meanings into the effects and associations of these variables. Values greater than 0.4 or -0.4 should be selected as related variables forming the particular factors.

The analysis extracted four factors with more than a $10 \%$ contribution ratio. Factor 1 ( $22.8 \%$ contribution ratio) comprised PR with a minus value, and FEV1.0\% and V25/H with positive values. This factor is assumed to represent respiratory factors and fewer PR cases showed higher (better) pulmonary function tests for obstructive changes. Factor 2 (17.7\% contribution ratio) comprised the titer indices of antiCENP-B and Scl-70 autoantibodies, IgG and age, all with plus values. This factor is understood as an immunological factor 
Table II. Results of factor analysis (varimax method).

\begin{tabular}{lccccc}
\hline & & \multicolumn{5}{c}{ Extracted factors } \\
\cline { 3 - 6 } Parameter & Unit & 1 & 2 & 3 & 4 \\
\hline CENP-B & Titer index & -0.026 & $\mathbf{0 . 7 7 3}$ & -0.255 & $\mathbf{- 0 . 4 0 5}$ \\
Scl-70 & Titer index & 0.194 & $\mathbf{0 . 4 5 9}$ & -0.207 & $\mathbf{0 . 4 8 9}$ \\
IgG & $\mu \mathrm{g} / \mathrm{ml}$ & 0.196 & $\mathbf{0 . 5 9 5}$ & 0.293 & 0.110 \\
IgA & $\mu \mathrm{g} / \mathrm{ml}$ & -0.043 & 0.001 & -0.045 & $\mathbf{0 . 9 2 8}$ \\
IgM & $\mu \mathrm{g} / \mathrm{ml}$ & 0.575 & 0.325 & -0.010 & -0.032 \\
Age & Years & 0.072 & $\mathbf{0 . 6 8 5}$ & -0.037 & 0.150 \\
PR & Grade & $\mathbf{- 0 . 4 3 2}$ & 0.082 & $\mathbf{0 . 6 9 8}$ & -0.002 \\
\%VC & $\%$ & 0.076 & -0.097 & $\mathbf{0 . 8 6 5}$ & -0.114 \\
FEV1.0 $(\%)$ & $\%$ & $\mathbf{0 . 8 8 0}$ & 0.130 & -0.318 & 0.017 \\
V25/H & $1 / \mathrm{sec} / \mathrm{m}$ & $\mathbf{0 . 9 4 7}$ & 0.033 & 0.092 & 0.072 \\
Contrib. rate & $\%$ & 22.8 & 17.7 & 15.4 & 13.2 \\
\hline
\end{tabular}

Factor analysis is a statistical method used to describe variability among observed, correlated variables in terms of a potentially lower number of unobserved variables called factors. In this study, it was required to assess whether or not the titer value of anti CENP-B as well as anti Scl-70 antibodies in SIL related with respiratory parameteress, or related with immunological parameters. Thus, five assumed respiratory parameters (age, PR, \%VC, FEV1.0 (\%) and $\mathrm{V} 25 / \mathrm{H}$ ) and three other immunological parameters (IgG, A and M) with above-mentioned titers of two auto-antibodies were applied to the factor analysis. Bold indicates parameters that had factor loading greater than \pm 0.4 . These parameters contributed significantly to the extraction of factors, positively or negatively. CENP-B, Centromere protein B; Scl-70, anti-topoisomerase; Ig, immunoglobulin; PR, profusion rate; \%VC, \% vital capacity; FEV1.0 (\%), forced expiratory volume $1.0(\mathrm{sec}) \%$; $\mathrm{V} 25 / \mathrm{H}$, forced expiratory flow at $25 \%$ vital capacity divided by body height $(\mathrm{m})$; Contrib. contribution.

with aged patients showing a tendency for higher antibodies and $\mathrm{IgG}$. The third factor is formed by PR and \% VC with a $15 \%$ contribution ratio. This factor is unusual for pathological consideration in SIL because it indicated that cases with worse radiological findings may have a better $\% \mathrm{VC}$. The forth factor with a $13.2 \%$ contribution ratio was formed by the titer index of anti-CENP-B autoantibody with a minus value, the anti-Scl-70 autoantibody with a plus value, as well as the IgA level with a plus value. As found in analyses of individual correlations, the titer index of anti-Scl-70 autoantibody and IgA showed a positive correlation. This forth factor indicated that even though the titer index of anti-Scl-70 autoantibody was located in the range of $\mathrm{HV}$, among these titers there is a correlation with $\operatorname{Ig} \mathrm{A}$ and this tendency was the opposite of that observed for the titer index of anti-CENP-B autoantibody.

\section{Discussion}

SIL cases include individuals suffering from pulmonary symptoms such as shortness of breath, respiratory infections such as tuberculosis, and chronic bronchitis due to chronic occupational inhalation of silica particles that cause pulmonary fibrosis $(1,2)$. In addition, the International Agency for Research on Cancer (IARC) certified that lung cancer is definitely caused by silica particles (3). Furthermore, SIL cases often complicate with autoimmune diseases (36-38). Caplan's syndrome is one of the well-known entities indicating SIL, as with rheumatoid arthritis (4-6). SSc is also a frequent complication shown by SIL (11-13). Recent reports have shown the association of SIL with ANCA-related vasculitis $(9,10)$. The mechanism of silica-induced dysregulation of autoimmunity is thought to involve silica acting as an adjuvant to existing autoantibodies to antigen-presenting cells (APC) and T cells (14-16).

We have been investigating the direct action of silica particles on immune competent cells and found that silica particles chronically activate both Tresp and Treg (23-25). These activations result in Tresp proceeding toward a longer survival to produce extracellular inhibitory molecules for CD95/Fas-mediated apoptosis such as soluble Fas, DcR3 and other alternatively spliced Fas variants (20-22). This population may include self-recognizing clones that cause the observed autoimmune diseases (17-19). On the other hand, there is another T-cell population that results in reduction of the expression of various intracellular molecules inhibiting Fas-mediated apoptosis physiologically such as I-Flice, sentrin and I-CAD (39). This population was thought to proceed easily towards apoptosis and is recruited from the bone marrow (39). Actually, Treg in SIL showed excess expression of the CD95/Fas molecule compared to that in HV (23). The chronic activation of Treg in SIL induces higher Fas expression, which results in the easy progression for Fas-mediated apoptosis in Treg that may be repeatedly recruited from bone marrow in SIL. However, these cellular events result in an unbalance of the Tresp (increase) and Treg (decrease) populations (23). The CD4+25+ fraction in SIL showed a reduction of Treg function due to contamination of chronically activated and CD25 expressing Tresp, and a decrease of the real Treg expressing the forkhead box P3 (FoxP3) master gene $(23,40)$.

In fact, SIL was positive for various autoantibodies such as anti-CD95/Fas (26), anti-caspase 8 (31), anti-desmoglein (30), as well as anti-Scl-70 antibodies (27-29). Moreover, our previous analyses showed the different distribution of HLA class II alleles in anti-topoisomerase I (Scl-70) autoantibody responders between silicosis and systemic sclerosis patients, with a common distinct amino acid sequence in the HLA-DQB1 domain (27-29).

The aim of this study is to evaluate and extract certain variables such as titer of anti-CENP-B antibody may indicate future dysregulation of autoimmune condition in SIL. Thus, titers such as anti-CENP-B and anti-Sci-70 auto antibodies are not required to be significantly higher than $\mathrm{HV}$ and similarly higher to SSc. In other word, as we consider that there may be SIL patients who progress toward immunological disorders rather than respiratory impairment (of course most of SIL may progress respiratory impairment rather than immunological disorders, or both pathological status progress paralleled), we hoped to find some parameters which indicate SIL predominantly progressing to immune status as we reported previously using soluble Fas (20) and serum sIL-2R (32). In this concept, it is not necessary that SIL with higher anti CENP-B titer is promised to progress toward SSc, however, of course they may develop SSC as well as other autoimmune diseases such as ANCA-related vasculitis and RA. In addition, all examined 
SIL were not revealed any symptoms or diagnostic criteria for various autoimmune diseases, especially patients with the highest anti-CENP-B was not complicated with SSc.

The clinical roles of anti-CENP-B and anti-Scl-70 autoantibody in SIL were examined in this study in regard to the uncommon detection of autoantibodies in SIL. Although no SIL were positive for anti-Scl-70 autoantibody, one case showed a sufficiently high titer index of anti-CENP-B autoantibody, and most SIL showed a tendency for a value higher than that in HV. In addition, if we assume an immune status of 1,2 and 3 for HV, SIL and SSc, respectively, we find a positive correlation between this assumed immune status and the whole titer index $\left(\log _{10}\right)$ of the anti-CENP-B autoantibody.

The results of analyses of individual correlations among various clinical parameters including respiratory and immunological items and the factor analysis indicated that the titer index of the anti-CENP-B autoantibody is not related to respiratory parameters. The increase of the titer index of anti-CENP-B autoantibody was then linked to the increase of the titer index of the anti-Scl-70 autoantibody, serum $\mathrm{IgG}$ and age. On the other hand, the titer indices of both autoantibodies indicated an opposite association with serum $\operatorname{IgA}$ values. The titer index of the anti-Scl-70 autoantibody was positively related with serum IgA and those of CENP-B indicated an opposite association. Thus, both autoantibodies may suggest a different immune disturbance in SIL, whereas all cases of SIL examined in this study basically showed a similar titer index for the anti-Scl-70 autoantibody compared with HV. This finding may require further analysis in future investigations, including cases of SIL positive for the anti-Scl-70 autoantibody.

The overall results show that the anti-CENP-B autoantibody was detected in SIL and the titer index of this autoantibody may indicate altered immune status in SIL. The titer index of the anti-CENP-B autoantibody, as well as serum soluble Fas (20) and serum sIL-2R (32), may be a biomarker for dysregulation in cases of SIL. Actually, we reported previously that there are some SIL who exhibited better respiratory conditions in association with a worse immunological status $(41,42)$. The future clinical follow-up of SIL may therefore need to consider respiratory and immunological aspects.

\section{Acknowledgements}

This study was supported by a KAKENHI grant from the Ministry of Education, Culture, Sports, Science and Technology of Japan (grant no. 25460825), a research grant from the Ryobi Teien MemorialFoundation, Kawasaki Medical School Research Grants (2012), a research grant from the Kawasaki Medical and Welfare Promoting Foundation (2012), and Kawasaki Medical School research grants (grant nos. 23S5, 24S6 and 26B16).

\section{References}

1. Rimal B, Greenberg AK and Rom WN: Basic pathogenetic mechanisms in silicosis: Current understanding. Curr Opin Pulm Med 11: 169-173, 2005.

2. Rees D and Murray J: Silica, silicosis and tuberculosis. Int J Tuberc Lung Dis 11: 474-484, 2007.

3. IARC Monograph on the Evaluation of Carcinogenic Risks to Humans. WHO, Geneva, 1997.

4. Caplan A: Rheumatoid disease and pneumoconiosis (Caplan's syndrome). Proc R Soc Med 52: 1111-1113, 1959.
5. Constantinidis K: Pneumoconiosis and Rheumatoid arthritis (Caplan's syndrome). Br J Clin Pract 31: 25-31, 1977.

6. Kroesen S, Itin PH and Hasler P: Arthritis and interstitial granulomatous dermatitis (Ackerman syndrome) with pulmonary silicosis. Semin Arthritis Rheum 32: 334-340, 2003.

7. Lucas CD, Amft N and Reid PT: Systemic lupus erythematosus complicating simple silicosis. Occup Med (Lond) 64: 387-390, 2014.

8. Yamazaki S, Yoshiike F, Hirai K, Kakegawa T, Ikeda M, Nagata A, Saito G, Nishimura H, Hosaka N and Ehara T: Silica-associated systemic lupus erythematosus in an elderly man. Intern Med 46: 1867-1871, 2007.

9. Saeki T, Fujita N, Kourakata H, Yamazaki H and Miyamura S: Two cases of hypertrophic pachymeningitis associated with myeloperoxidase antineutrophil cytoplasmic autoantibody (MPO-ANCA)-positive pulmonary silicosis in tunnel workers. Clin Rheumatol 23: 76-80, 2004.

10. Tervaert JW, Stegeman CA and Kallenberg CG: Silicon exposure and vasculitis. Curr Opin Rheumatol 10: 12-17, 1998.

11. Rodnan GP, Benedek TG, Medsger TA Jr and Cammarata RJ: The association of progressive systemic sclerosis (scleroderma) with coal miners' pneumoconiosis and other forms of silicosis. Ann Intern Med 66: 323-334, 1967.

12. Ben Abdelghani K, Fazaa A, Souabni L and Zakraoui L: Association of pulmonary silicosis and systemic sclerosis. BMJ Case Rep 2015: pii, 2015.

13. Sluis-Cremer GK, Hessel PA, Nizdo EH, Churchill AR and Zeiss EA: Silica, silicosis, and progressive systemic sclerosis. Br J Ind Med 42: 838-843, 1985.

14. Vera-Lastra O, Medina G, Cruz-Dominguez Mdel P, Jara LJ and Shoenfeld Y: Autoimmune/inflammatory syndrome induced by adjuvants (Shoenfeld's syndrome): Clinical and immunological spectrum. Expert Rev Clin Immunol 9: 361-373, 2013.

15. Perricone C, Colafrancesco S, Mazor RD, Soriano A, Agmon-Levin N and Shoenfeld Y: Autoimmune/inflammatory syndrome induced by adjuvants (ASIA): Unveiling the pathogenic, clinical and diagnostic aspects. J Autoimmun 47: $1-16,2013$

16. Parks CG, Conrad K and Cooper GS: Occupational exposure to crystalline silica and autoimmune disease. Environ Health Perspect 107 (Suppl 5): S793-S802, 1999.

17. Otsuki T, Miura Y, Nishimura Y, Hyodoh F, Takata A, Kusaka M, Katsuyama H, Tomita M, Ueki A and Kishimoto T: Alterations of fas and fas-related molecules in patients with silicosis. Exp Biol Med (Maywood) 231: 522-533, 2006.

18. Otsuki T, Maeda M, Murakami S, Hayashi H, Miura Y, Kusaka M, Nakano T, Fukuoka K, Kishimoto T, Hyodoh F, et al: Immunological effects of silica and asbestos. Cell Mol Immunol 4: 261-268, 2007.

19. Maeda M, Nishimura Y, Kumagai N, Hayashi H, Hatayama T, Katoh M, Miyahara N, Yamamoto S, Hirastuka J and Otsuki T: Dysregulation of the immune system caused by silica and asbestos. J Immunotoxicol 7: 268-278, 2010.

20. Tomokuni A, Aikoh T, Matsuki T, Isozaki Y, Otsuki T, Kita S, Ueki H, Kusaka M, Kishimoto T and Ueki A: Elevated soluble Fas/APO-1 (CD95) levels in silicosis patients without clinical symptoms of autoimmune diseases or malignant tumours. Clin Exp Immunol 110: 303-309, 1997.

21. Otsuki T, Tomokuni A, Sakaguchi H, Aikoh T, Matsuki T, Isozaki Y, Hyodoh F, Ueki H, Kusaka M, Kita S and Ueki A: Over-expression of the decoy receptor 3 (DcR3) gene in peripheral blood mononuclear cells (PBMC) derived from silicosis patients. Clin Exp Immunol 119: 323-327, 2000.

22. Otsuki T, Sakaguchi H, Tomokuni A, Aikoh T, Matsuki T, Isozaki Y, Hyodoh F, Kawakami Y, Kusaka M, Kita S and Ueki A: Detection of alternatively spliced variant messages of fas gene and mutational screening of fas and fas ligand coding regions in peripheral blood mononuclear cells derived from silicosis patients. Immunol Lett 72: 137-143, 2000.

23. Hayashi H, Miura Y, Maeda M, Murakami S, Kumagai N, Nishimura Y, Kusaka M, Urakami K, Fujimoto W and Otsuki T: Reductive alteration of the regulatory function of the CD4(+)CD25(+) T cell fraction in silicosis patients. Int $\mathbf{J}$ Immunopathol Pharmacol 23: 1099-1109, 2010.

24. Lee S, Hayashi H, Maeda M, Chen Y, Matsuzaki H, Takei-Kumagai N, Nishimura Y, Fujimoto W and Otsuki T: Environmental factors producing autoimmune dysregulation-chronic activation of $\mathrm{T}$ cells caused by silica exposure. Immunobiology 217: 743-748, 2012. 
25. Lee S, Matsuzaki H, Kumagai-Takei N, Yoshitome K, Maeda M, Chen Y, Kusaka M, Urakami K, Hayashi H, Fujimoto W, et al: Silica exposure and altered regulation of autoimmunity. Environ Health Prev Med 19: 322-329, 2014.

26. Takata-Tomokuni A, Ueki A, Shiwa M, Isozaki Y, Hatayama T, Katsuyama H, Hyodoh F, Fujimoto W, Ueki H, Kusaka M, et al: Detection, epitope-mapping and function of anti-fas autoantibody in patients with silicosis. Immunology 116: 21-29, 2005.

27. Ueki A, Isozaki Y, Tomokuni A, Otsuki T, Hydoh F, Sakaguchi H, Tanaka S and Kusaka M: Is the anti-topoisomerase I autoantibody response associated with a distinct amino acid sequence in the HLA-DQbeta1 domain? Arthritis Rheum 44: 491-492, 2001.

28. Ueki A, Isozaki Y, Tomokuni A, Tanaka S, Otsuki T, Kishimoto T, Kusaka M, Aikoh T, Sakaguchi H and Hydoh F: Autoantibodies detectable in the sera of silicosis patients. The relationship between the anti-topoisomerase I antibody response and HLA-DQB1*0402 allele in Japanese silicosis patients. Sci Total Environ 270: 141-148, 2001.

29. Ueki A, Isozaki Y, Tomokuni A, Ueki H, Kusaka M, Tanaka S, Otsuki T, Sakaguchi H and Hyodoh F: Different distribution of HLA class II alleles in anti-topoisomerase I autoantibody responders between silicosis and systemic sclerosis patients, with a common distinct amino acid sequence in the HLA-DQB1 domain. Immunobiology 204: 458-465, 2001

30. Ueki H, Kohda M, Nobutoh T, Yamaguchi M, Omori K, Miyashita Y, Hashimoto T, Komai A, Tomokuni A and Ueki A: Antidesmoglein autoantibodies in silicosis patients with no bullous diseases. Dermatology 202: 16-21, 2001.

31. Ueki A, Isozaki Y, Tomokuni A, Hatayama T, Ueki H, Kusaka M, Shiwa M, Arikuni H, Takeshita T and Morimoto M: Intramolecular epitope spreading among anti-caspase-8 autoantibodies in patients with silicosis, systemic sclerosis and systemic lupus erythematosus, as well as in healthy individuals. Clin Exp Immunol 129: 556-561, 2002.

32. Hayashi H, Maeda M, Murakami S, Kumagai N, Chen Y, Hatayama T, Katoh M, Miyahara N, Yamamoto S, Yoshida Y, et al: Soluble interleukin-2 receptor as an indicator of immunological disturbance found in silicosis patients. Int J Immunopathol Pharmacol 22: 53-62, 2009.
33. International Labour Organization (ILO): ILO International Classification of Radiographs of Pneumoconioses. Revised edition 2000. ILO, Geneva, 2000.

34. Kallenberg CG: Early detection of connective tissue disease in patients with Raynaud's phenomenon. Rheum Dis Clin North Am 16: 11-30, 1990.

35. Basu D and Reveille JD: Anti-scl-70. Autoimmunity 38: 65-72, 2005

36. Benedek TG: Rheumatoid pneumoconiosis. Documentation of onset and pathogenic considerations. Am J Med 55: 515-524, 1973.

37. Uber CL and McReynolds RA: Immunotoxicology of silica. Crit Rev Toxicol 10: 303-319, 1982

38. Steenland K and Goldsmith DF: Silica exposure and autoimmune diseases. Am J Ind Med 28: 603-608, 1995.

39. Otsuki T, Tomokuni A, Sakaguchi H, Hyodoh F, Kusaka M and Ueki A. Reduced expression of the inhibitory genes for Fas-mediated apoptosis in silicosis patients. J Occup Health 42: 163-168, 2000.

40. Wu P, Miura Y, Hyodoh F, Nishimura Y, Hatayama T, Hatada S, Sakaguchi H, Kusaka M, Katsuyama H, Tomita M and Otsuki T: Reduced function of CD4+25+ regulatory $\mathrm{T}$ cell fraction in silicosis patients. Int $\mathrm{J}$ Immunopathol Pharmacol 19: 357-368, 2006.

41. Otsuki T, Ichihara K, Tomokuni A, Sakaguchi H, Aikoh T, Matsuki T, Isozaki Y, Hyodoh F, Kusaka M, Kita S and Ueki A: Evaluation of cases with silicosis using the parameters related to fas-mediated apoptosis. Int J Mol Med 4: 407-411, 1999.

42. Otsuki T, Matsuzaki H, Lee S, Kumagai-Takei N, Yamamoto S, Hatayama T, Yoshitome K and Nishimura Y: Environmental factors and human health: Fibrous and particulate substance-induced immunological disorders and construction of a health-promoting living environment. Environ Health Prev Med 21: 71-81, 2016. 